\title{
Invertibility of Generalized Space-Time Autoregressive Model with Random Weight
}

\author{
Yundari' ${ }^{1}$, Setyo Wira Rizki² \\ ${ }^{1}$ Mathematics Department, Faculty of Mathematics and Natural Science, Universitas \\ Tanjungpura Pontianak, Indonesia \\ ${ }^{2}$ Statistics Department, Faculty of Mathematics and Natural Science, Universitas \\ Tanjungpura Pontianak, Indonesia \\ Email: yundari@math.untan.ac.id
}

\begin{abstract}
The generalized linear process accomplishes stationarity and invertibility properties. The invertibility property must be having a series of convergence conditions of the process parameter. The generalized Space-Time Autoregressive (GSTAR) model is one of the stationary linear models therefore it is necessary to reveal the invertibility through the convergence of the parameter series. This article studies the invertibility of model GSTAR(1;1) with kernel random weight. The result shows that the model GSTAR(1;1) under kernel random weight fulfills the invertibility property and obtains a finite order of Generalized Space-Time Moving Average (GSTMA) process. The other result obtained is the time order of the finite orde $7<n<30$. On the Triangular kernel resulted in the relatively great value $n$, so that it does not apply to the kernel with a finite value $n$. The GSTAR(1;1) model with random kernel weight is applied to the data of tea production in six plantantion area in West Java. The RMSE value of data estimation obtained is quite small. It follows the original data pattern at each research location respectively.
\end{abstract}

Keywords: autoregressive process; generalized linear process; invertibility; stationarity

\section{INTRODUCTION}

Theoretically, the first order of the autoregressive model, AR(1), of the univariate time series is equivalent to the moving average model with infinity order, $\operatorname{MA}(\infty)$ [1]. It happens to the multivariate model that the vector autoregressive model, VAR(1), is comparable with the model $\operatorname{VMA}(\infty)$ [2]. These properties are known as the invertibility property of the autoregression model orde 1 . The GSTAR(1;1) model is a member of the autoregression model family [3]. The question of research, Is the GSTAR(1;1) model also equivalent to the GSTMA $(\infty ; 1)$ model? The theoretical study of the GSTAR model has be done in [4] about the model stationarity using the inverse of the autocorrelation matrix. Furthermore, [5] tells about the estimation property of the parameter GSTAR using the least square method, observes the error assumption of the model GSTAR [6] and the GSTAR containing outlier [7]. Also, the development of the GSTAR model has been carried out by several researchers such as GSTAR-GARCH [8], GSTAR-SUR [9], GSTAR-Kriging [10] and others. 
Several researchers have developed the spatial weight matrix determination, such as [11] using a uniform spatial weight matrix namely the closest neighbors are given the same weight. [5] uses a binary weight matrix considering the uniform weight as a comparison. The weight matrix determination using cross-correlation have also be done by [12]. All of the researchers use distance as the basis of the weight matrix determination. [13] proposes a Fuzzy set approach based on observational data in determining the weight matrix, but the approach still produces the weights assigned is not random. Determining random weight matrix have be be done by author by using some kernel functions approach [3]. Furthermore, the spatial weight effect of the random kernel is also examined for its stationary properties [14]. Some of the space-time data applied using the GSTAR model are the tourist number data at several tourist attractions [15], the tea production data [5], the GDP data in the countries in Europe [11], the chili prices prediction [16], the data of log Gamma Ray [3], the rainfall data [10] and so on.

This paper discusses the GSTAR model with a random weight using the kernel function. The kernel function used is uniform, triangular, Epanechnikov, cosine dan Gaussian. The kernel functions present the constant function, linear, square, cosine, dan exponential. Moreover, the research talks about the weight matrix effect of kernel spatial to its invertibility. In notation, the weight matrix using the kernel function is denoted by $\widetilde{\mathbf{W}}=\left(\tilde{W}_{i j}\right)$ and the parameter matrix $\operatorname{GSTAR}(1 ; 1)$ is represented by $\widetilde{\boldsymbol{\Phi}}$. Besides, the study discloses the convergence of each kernel function to its invertibility. The article begins with the invertibility theory of the AR(1) model and VAR(1). The next section explains the kernel function and continued with the study of the GSTAR(1;1) model under the kernel weight. Both results and discussion will be conferred in the next section about the invertibility of the GSTAR model under both the kernel weight and the convergence to determine the order of the GSTMA model. In the last section, the paper implements the GSTAR(1;1) model with the Gaussian kernel weight on the tea production data in the six plantation area in West Java.

\section{METHODS}

This section will discuss the theories underlying the research namely the AR(1) model and VAR(1) which is the basis of the GSTAR(1;1) model formation. After that, the research studies the properties of each invertibility. The last, it will have conversed about the kernel function used to form the spatial weight matrix of the GSTAR(1;1) model.

\section{Invertibility of AR(1) and VAR(1) Process}

The autoregression process $(\mathrm{AR}(\mathrm{p}))$ is defined as below [1]:

$$
Y_{t}=\phi_{1} Y_{t-1}+\ldots+\phi_{p} Y_{t-p}+a_{t}
$$

with $\phi_{1}, \phi_{2}, \ldots, \phi_{p}$ are the autoregression parameters and $a_{t}$ is white noise process with mean is zero and variance is $\sigma_{a}^{2}$. If $p=1$ then it will be known as process AR(1), which is formulated as:

$$
Y_{t}=\phi Y_{t-1}+a_{t} \text {. }
$$

Besides, the process AR(1) is one of stationer linear models under a stationarity condition $|\phi|<1$, model AR(1) has the invertibility property such as:

$$
\begin{aligned}
& Y_{t}=\phi Y_{t-1}+a_{t} \\
& Y_{t}=\cdots+\phi^{k} a_{t-k}+\phi^{k-1} a_{t-(k-1)}+\cdots+\phi^{2} a_{t-2}+\phi a_{t-1}+a_{t}
\end{aligned}
$$


The last model obtained is the $\mathrm{MA}(\infty)$ model and the MA model surely stationer with $|\phi|<1$ and over convergence process. It results in the $\operatorname{AR}(1)$ is invertible to $\operatorname{AR}(1) \approx \operatorname{MA}($ $\infty)$.

On the process $A R(1)$, it considers one random variable with some times. If the observation is worked by using several random variables which each of them through the process AR(1) so it is known as the first order of the vector of autoregressive (VAR(1)). The model VAR(1) can be framed as follows:

$$
\mathbf{Y}_{t}=\phi \mathbf{Y}_{t-1}+\vec{a}_{t}
$$

The necessary and sufficient condition of stationarity of the VAR(1) is a solution of $\left|I_{k}-\phi_{1} B\right|=0$ less than one. The process VAR(1) can also be represented in the vector of moving average (VMA) or in the other words satisfied the invertibility property [2].

\section{The Kernel Function}

The continuous real function $f: R \rightarrow R$ is denoted as the kernel function if satisfied the sum of integral is one, symmetrically for each $x \in R$, the mean equal to zero and the finite variance. An example of the kernel function along with its efficiency properties which learned in Table 1. The notation $R(\mathrm{k})=\int\{k(x)\}^{2} d x$ states "roughness" of the kernel function $k$. The notation $\sigma_{K}^{2}=\int x^{2} k(x) d x$ is a variance of the kernel function, while the efficiency of the kernel function is obtained from $\left\{C\left(K^{*}\right) / C(\mathrm{~K})\right\}^{5 / 4}=1$, where $C(K)=\left\{R(K)^{4}\left(\sigma_{K}^{2}\right)^{2}\right\}^{1 / 5}$.

Table 1. The shape of kernel function and its properties. The bound of its domain between -1 and 1 (and 0 for outside the domain), except for the Gaussian kernel is applicable for all the real numbers [17].

\begin{tabular}{|c|c|c|c|c|c|}
\hline The Kernel & $\begin{array}{l}\text { The Form of } \\
\text { Function }\end{array}$ & $R(k)$ & $\sigma_{K}^{2}$ & $\begin{array}{c}\text { The } \\
\text { Efficiency }\end{array}$ & $\begin{array}{c}\text { The } \\
\text { Domain }\end{array}$ \\
\hline \multirow{2}{*}{$\begin{array}{c}\text { Uniform } \\
\text { (Seragam kernel) }\end{array}$} & \multirow[t]{2}{*}{$k(x)=1 / 2$} & 1 & 1 & \multirow[t]{2}{*}{0.9295} & \multirow[t]{2}{*}{$(-1,1)$} \\
\hline & & $\overline{2}$ & $\overline{3}$ & & \\
\hline \multirow[t]{2}{*}{ Triangular } & \multirow{2}{*}{$k(x)=1-|x|$} & $\underline{2}$ & 1 & \multirow[t]{2}{*}{0.9859} & \multirow[t]{2}{*}{$(-1,1)$} \\
\hline & & 3 & 6 & & \\
\hline \multirow[t]{2}{*}{ Epanechnikov } & \multirow{2}{*}{$k(x)=\frac{3}{4}\left(1-x^{2}\right)$} & $\underline{3}$ & 1 & \multirow[t]{2}{*}{1} & \multirow[t]{2}{*}{$(-1,1)$} \\
\hline & & $\overline{5}$ & $\overline{5}$ & & \\
\hline \multirow[t]{2}{*}{ Cosinus } & $\pi \quad(\pi)$ & $\pi^{2}$ & \multirow{2}{*}{$1-\frac{8}{\pi^{2}}$} & \multirow[t]{2}{*}{0.9897} & \multirow[t]{2}{*}{$(-1,1)$} \\
\hline & $k(x)=\overline{4} \cos (\overline{2})$ & $\overline{16}$ & & & \\
\hline Gaussian & $k(x)=\frac{1}{\sqrt{2 \pi}} \exp \left\{-\frac{x^{2}}{2}\right\}$ & $\frac{1}{2 \sqrt{\pi}}$ & 1 & 0.9512 & $\mathbb{R}$ \\
\hline
\end{tabular}

The approximation of the kernel function as the weight function is generally used to estimate density and regression function. The procedure of the kernel function is the sum of some kernel function to each point corresponded to every surrounding point (see Figure 1). In general, the kernel function of a point linked to it's the nearest point, for 
example, $x$ and $y$ are $k\left(\frac{x-y}{h}\right)$. The notation $h$ is a bandwidth controlling smoothness of the kernel function.

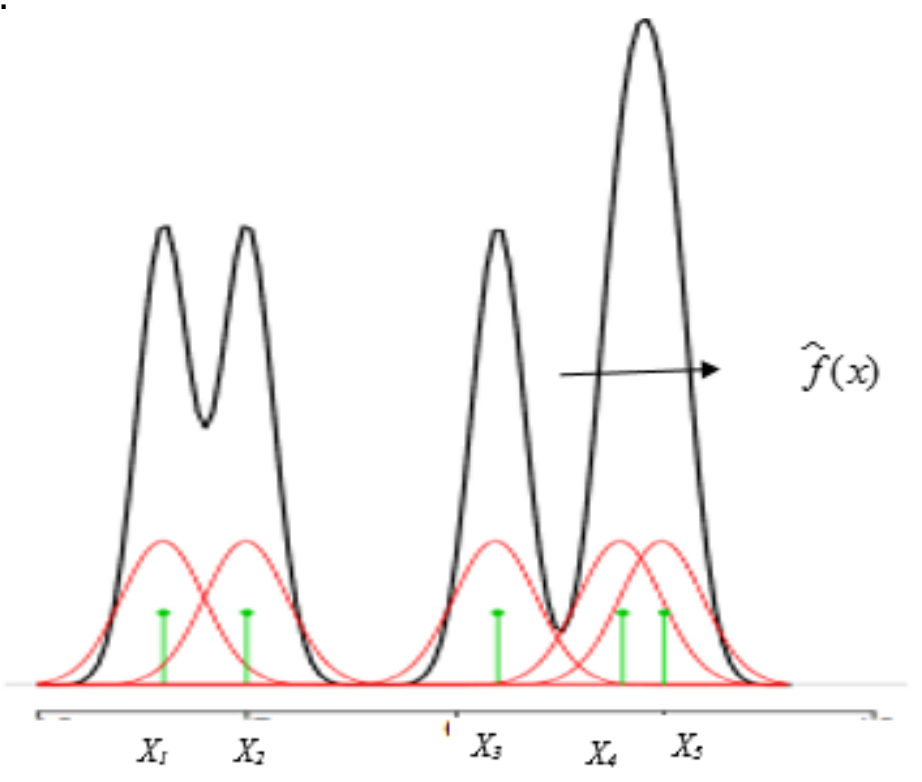

Figure 1. The plot of kernel density estimation. If there are a lot of observations which is close to point $\mathrm{x}$ then $f(x)$ has great value. On other hand, if there are less $X_{i}$ closed to the point $\mathrm{x}$ then $f(x)$ has a small value.

\section{The GSTAR(1;1) Model with the kernel Weight}

The novel method to determine the spatial weight matrix of the model GSTAR recommended is by using the kernel function. Kernel location weight is attained by adopting the kernel estimator of Nadaraya-Watson [18] and using an average value of the observation on every single location $\bar{Y}_{i}$.

Average value selection of observation in each location is intended to find overall data property (data centering) by ignoring outlier of an observation data.

Centralization process $\left\{Y_{i}(t)\right\}$ following a model GSTAR $(1 ; 1)$ kernel weight is written as:

$$
Y_{i}(t)=\phi_{0 i} Y_{i}(t-1)+\phi_{1 i} \sum_{j=1}^{N} \widetilde{W}_{i j} Y_{j}(t-1)+\varepsilon_{i}(t), t=1, \ldots, T, i=1, \ldots, N
$$

This model has a spatial weight

$$
\tilde{W}_{i j}=\frac{k\left(\frac{\bar{Y}_{i}-\bar{Y}_{j}}{h}\right)}{\sum_{\substack{\ell=1 \\ \ell \neq i}}^{N} k\left(\frac{\bar{Y}_{i}-\bar{Y}_{\ell}}{h}\right)},
$$

with notation $k($.$) is the kernel function, h$ represents a smoother parameter of the kernel function $k$ and $Y_{i}(t)$ declares an observation on-time $t$ at location $i$. The term weight matrix can be written as, 


$$
\widetilde{\mathbf{W}}=\left[\begin{array}{cccc}
0 & \widetilde{W}_{12} & \cdots & \widetilde{W}_{1 N} \\
\widetilde{W}_{21} & 0 & \cdots & \widetilde{W}_{2 N} \\
\vdots & \vdots & \ddots & \vdots \\
\widetilde{W}_{N 1} & \widetilde{W}_{N 2} & \cdots & 0
\end{array}\right]
$$

The result of the weight matrix $\widetilde{\mathbf{W}}$, by the kernel function approach, appears to satisfy the properties of the random weight matrix. It is caused by the weight that originated from the random variable data. It is the observation data and fulfilled the property $\sum_{j=1}^{N} W_{i j}=1$, $N>1$.

After obtaining the matrix of kernel spatial weight, the parameter estimation of the GSTAR $(1 ; 1)$ model is carried out using the least squares method followed by validating model. The model validating is held by doing 2 steps namely the parameter significance test and the residual test. The parameter significance test uses the parameter matrix eigen value of the GSTAR(1;1) model and the residual test using the plot of data error (error randomness) and the QQ plot of error (normality).

\section{RESULTS AND DISCUSSION}

The symbol writing of parameter matrix for the $\operatorname{GSTAR}(1 ; 1)$ model based on Equation (1) to $\boldsymbol{\Phi}_{0}=\operatorname{diag}\left(\phi_{01}, \cdots, \phi_{0 N}\right), \boldsymbol{\Phi}_{1}=\operatorname{diag}\left(\phi_{11}, \cdots, \phi_{1 N}\right)$ dan $\tilde{\boldsymbol{W}}_{=}\left(w_{i j}\right)$, so that the model GSTAR $(1 ; 1)$ can be expressed in the matrix as follows

$$
\begin{aligned}
& \mathbf{Y}(t)=\boldsymbol{\Phi}_{\mathbf{0}} \mathbf{Y}(t-1)+\boldsymbol{\Phi}_{\mathbf{1}} \widetilde{\mathbf{W}} \mathbf{Y}(t-1)+\boldsymbol{\varepsilon}(t) \\
& \mathbf{Y}(t)=\left(\boldsymbol{\Phi}_{\mathbf{0}}+\boldsymbol{\Phi}_{\mathbf{1}} \widetilde{\mathbf{W}}\right) \mathbf{Y}(t-1)+\boldsymbol{\varepsilon}(t) .
\end{aligned}
$$

Representation of the GSTMA model from the GSTAR(1;1) model as below,

$$
\begin{aligned}
\mathbf{Y}(t) & =\left(\boldsymbol{\Phi}_{\mathbf{0}}+\boldsymbol{\Phi}_{\mathbf{1}} \widetilde{\mathbf{W}}\right) \mathbf{Y}(t-1)+\boldsymbol{\varepsilon}(t) \\
& =\left(\boldsymbol{\Phi}_{\mathbf{0}}+\boldsymbol{\Phi}_{\mathbf{1}} \widetilde{\mathbf{W}}\right)\left\{\left(\boldsymbol{\Phi}_{\mathbf{0}}+\boldsymbol{\Phi}_{\mathbf{1}} \widetilde{\mathbf{W}}\right) \mathbf{Y}(t-2)+\boldsymbol{\varepsilon}(t-1)\right\}+\boldsymbol{\varepsilon}(t) \\
& =\left(\boldsymbol{\Phi}_{\mathbf{0}}+\boldsymbol{\Phi}_{\mathbf{1}} \widetilde{\mathbf{W}}\right)^{2} \mathbf{Y}(t-2)+\left(\boldsymbol{\Phi}_{\mathbf{0}}+\boldsymbol{\Phi}_{\mathbf{1}} \widetilde{\mathbf{W}}\right) \boldsymbol{\varepsilon}(t-1)+\boldsymbol{\varepsilon}(t) \\
& =\boldsymbol{\varepsilon}(t)+\left(\boldsymbol{\Phi}_{\mathbf{0}}+\boldsymbol{\Phi}_{\mathbf{1}} \widetilde{\mathbf{W}}\right) \boldsymbol{\varepsilon}(t-1)+\left(\boldsymbol{\Phi}_{\mathbf{0}}+\boldsymbol{\Phi}_{\mathbf{1}} \widetilde{\mathbf{W}}\right)^{2} \mathbf{Y}(t-2) \\
& \vdots \\
& =\boldsymbol{\varepsilon}(t)+\left(\boldsymbol{\Phi}_{\mathbf{0}}+\boldsymbol{\Phi}_{\mathbf{1}} \widetilde{\mathbf{W}}\right) \boldsymbol{\varepsilon}(t-1)+\left(\boldsymbol{\Phi}_{\mathbf{0}}+\boldsymbol{\Phi}_{\mathbf{1}} \widetilde{\mathbf{W}}\right)^{2} \boldsymbol{\varepsilon}(t-2)+\cdots \\
& =\sum_{i=0}^{\infty} \widetilde{\boldsymbol{\Phi}}^{i} \boldsymbol{\varepsilon}(t-i)
\end{aligned}
$$

with $\widetilde{\boldsymbol{\Phi}}=\left(\boldsymbol{\Phi}_{0}+\Phi_{1} \widetilde{\mathbf{W}}\right)$.

For the GSTAR(1;1) model which is stationer, all of the eigenvalues $\widetilde{\boldsymbol{\Phi}}$ are between -1 and 1 so that $\widetilde{\boldsymbol{\Phi}}^{i} \rightarrow 0$ for $i \rightarrow \infty$. This is stated in Theorem 1 . 
Theorem 1. If $\widetilde{\boldsymbol{\Phi}}=\left(\boldsymbol{\Phi}_{\mathbf{0}}+\boldsymbol{\Phi}_{\mathbf{1}} \widetilde{\mathbf{W}}\right)$, and eigenvalue of $\widetilde{\boldsymbol{\Phi}}$ is between -1 dan 1 so $\lim _{n \rightarrow \infty} \widetilde{\boldsymbol{\Phi}}^{n}=\mathbf{0}$, for $n=0,1,2, \ldots$.

Proof: The matrix $\widetilde{\boldsymbol{\Phi}} \cdot \widetilde{\boldsymbol{\Phi}}$ is positive definite so that the matrix $\widetilde{\boldsymbol{\Phi}} \in \mathrm{C}^{k \times \ell}$ can be stated by the singular value decomposition (SVD), i.e a diagonal matrix $\mathbf{D} \in \mathrm{R}^{r \times r}, r \leq \min \{k, \ell\}$ and matrix $\mathbf{U} \in \mathrm{C}^{k \times k}, \mathbf{V} \in \mathrm{C}^{\ell \times \ell}$, so that

$$
\widetilde{\boldsymbol{\Phi}}=\mathbf{U D V} \quad \Leftrightarrow \quad \widetilde{\boldsymbol{\Phi}}^{\mathbf{n}}=\mathbf{U} \mathbf{D}^{\mathrm{n}} \mathbf{V}
$$

Because of matrix elements, $\mathbf{D}$ is a root of the eigenvalue of matrix $\widetilde{\boldsymbol{\Phi}}$ and eigenvalue of $\widetilde{\boldsymbol{\Phi}}$ is between -1 dan 1 so $\lim _{n \rightarrow \infty} \mathbf{D}^{n}=\mathbf{0}$. It resulted $\lim _{n \rightarrow \infty} \widetilde{\boldsymbol{\Phi}}^{n}=\mathbf{0}$.

This caused the invertibility property of the GSTAR(1;1) model satisfied because of the coefficient of process $\{\mathbf{Y}(t-i)\}$ limits to zero. It confirms that $\operatorname{GSTAR}(1 ; 1) \simeq \operatorname{GSTMA}(\infty ; 1)$. The orde determination of GSTMA is theoretically done by considering the convergence level of every kernel function used. If it is reviewed from every viewpoint of the kernel function, the limit value approaching zero (Table 2).

The invertibility property stated that $\operatorname{GSTAR}(1 ; 1) \simeq \operatorname{GSTMA}(\infty ; 1)$. In statistics, the orde of time GSTMA on the $\operatorname{GSTMA}(\infty ; 1)$ model does not mean infinite, but it can be determined by a finite order such as $n$. It is stated in Theorem 2 .

Table 2. The limit result of each kernel function. It seems that the overall kernel function having a limit value is zero.

\begin{tabular}{cc}
\hline The Kernel & $\begin{array}{c}\text { The limit result of the } \\
\text { function }\end{array}$ \\
\hline The Uniform & $\lim _{n \rightarrow \infty}\left(\frac{1}{2}\right)^{n}=0$ \\
The Triangular & $\lim _{n \rightarrow \infty}((1-|x|))^{n}=0$ \\
The Epanechnikov & $\lim _{n \rightarrow \infty}\left(\frac{3}{4}\left(1-x^{2}\right)\right)^{n}=0$ \\
The Cosinus & $\lim _{n \rightarrow \infty}\left(\frac{\pi}{4} \cos \left(\frac{\pi}{2} x\right)\right)^{n}=0$ \\
The Gaussian & $\lim _{n \rightarrow \infty}\left(\frac{1}{\sqrt{2 \pi}} \exp \left\{-\frac{x^{2}}{2}\right\}\right)^{n}=0$ \\
\hline
\end{tabular}

Theorem 2. If given a process $\left\{Y_{i}(t)\right\}$ following the model GSTAR(1;1) with a weight matrix of kernel spatial and satisfied the invertibility property so that $\operatorname{GSTAR}(1 ; 1) \simeq \operatorname{GSTMA}(\infty ; 1)$ then

$$
\left\|\widetilde{\boldsymbol{\Phi}} \mathbf{Y}(t-1)+\boldsymbol{\varepsilon}(t)-\sum_{i=0}^{n} \widetilde{\boldsymbol{\Phi}}^{i} \boldsymbol{\varepsilon}(t-i)\right\| \rightarrow 0 \text {, for } n \rightarrow \infty .
$$


Proof: Given the GSTAR(1;1) model and the GSTMA( $\infty, 1)$ model, with the help of matrix norm from the difference of both equivalent models, obtained

$$
\begin{aligned}
& \left\|\widetilde{\mathbf{\Phi}} \mathbf{Y}(t-1)+\boldsymbol{\varepsilon}(t)-\sum_{i=0}^{n} \widetilde{\boldsymbol{\Phi}}^{i} \boldsymbol{\varepsilon}(t-i)\right\|=\mid \widetilde{\mathbf{\Phi}} \mathbf{Y}(t-1)-\sum_{i=1}^{n} \widetilde{\mathbf{\Phi}}^{i} \boldsymbol{\varepsilon}(t-i) \| \\
& =\left\|\widetilde{\mathbf{\Phi}} \mathbf{Y}(t-1)-\sum_{i=1}^{n} \widetilde{\mathbf{\Phi}}^{i}(\mathbf{Y}(t-i)-\widetilde{\mathbf{\Phi}} \mathbf{Y}(t-i-1))\right\| \\
& =\left\|\widetilde{\mathbf{\Phi}} \mathbf{Y}(t-1)-\widetilde{\mathbf{\Phi}} \mathbf{Y}(t-1)+\widetilde{\boldsymbol{\Phi}}^{2} \mathbf{Y}(t-2)-\sum_{i=2}^{n} \widetilde{\mathbf{\Phi}}^{i}(\mathbf{Y}(t-i)-\widetilde{\mathbf{\Phi}} \mathbf{Y}(t-i-1))\right\| \\
& =\| \widetilde{\mathbf{\Phi}}^{2} \mathbf{Y}(t-2)-\widetilde{\boldsymbol{\Phi}}^{2} \mathbf{Y}(t-2)-\sum_{i=3}^{n} \widetilde{\boldsymbol{\Phi}}^{i}(\mathbf{Y}(t-i)-\widetilde{\mathbf{\Phi}} \mathbf{Y}(t-i-1)) \mid \\
& =\left\|\widetilde{\mathbf{\Phi}}^{n} \mathbf{Y}(t-n)\right\|
\end{aligned}
$$

Furthermore, by using the property of matrix norm, it can be written as,

$$
\left\|\tilde{\boldsymbol{\Phi}}^{n} \mathbf{Y}(t-n)\right\| \leq\left\|\tilde{\boldsymbol{\Phi}}^{n}\right\|\|\mathbf{Y}(t-n)\| \leq c\left\|\tilde{\boldsymbol{\Phi}}^{n}\right\| \leq c\|\tilde{\boldsymbol{\Phi}}\|^{n}, \text { for a constant } c \in \mathbb{R} .
$$

It is defined previously that $\widetilde{\boldsymbol{\Phi}}=\Phi_{0}+\Phi_{1} \widetilde{\mathbf{W}}$ so

$$
\left\|\tilde{\mathbf{\Phi}}^{n}\right\|=\left\|\left(\Phi_{0}+\Phi_{1} \tilde{\mathbf{W}}\right)^{n}\right\| \leq\left\|\Phi_{0}\right\|^{n}+\left\|\Phi_{1}\right\|^{n}\|\tilde{\mathbf{W}}\|^{n}
$$

The value of diagonal matrix elements $\boldsymbol{\Phi}_{i}$ is between -1 and 1 , so that $\left\|\boldsymbol{\Phi}_{0}{ }^{n}\right\| \leq\left\|\boldsymbol{\Phi}_{0}\right\|^{n}=\left(\operatorname{maks} \sum_{i}\left|\phi_{0 i}\right|\right)^{n}=\left(a_{0}\right)^{n}$ for $0<a_{0}<1$. Similar to the parameter AR concerning location that is $\left\|\boldsymbol{\Phi}_{1}{ }^{n}\right\| \leq\left\|\boldsymbol{\Phi}_{1}\right\|^{n}=\left(\operatorname{maks} \sum_{i}\left|\phi_{1 i}\right|\right)^{n}=\left(b_{0}\right)^{n}$ for $0<b_{0}<1$. It results in Equation (2) being:

$$
\left\|\widetilde{\boldsymbol{\Phi}}^{n}\right\| \leq a_{0}{ }^{n}+b_{0}{ }^{n}\|\widetilde{\mathbf{W}}\|^{n}
$$

The matrix $\mathbf{W}$ is a spatial weight matrix and obtained through the kernel function. For each, the kernel function converges to zero (Table 2) and $\underset{n \rightarrow \infty}{\lim _{n} a_{0}{ }^{n}=0} \operatorname{limb}_{n \rightarrow \infty}{ }^{n}=0$. By using norm $\ell_{\infty}$ on matrix [19] is $\|\widetilde{\mathbf{W}}\|_{\infty}=\operatorname{maks}_{1 \leq i, j \leq n}\left|\widetilde{W}_{i j}\right|$, therefore that can be attained the norm of rank $n$ of the spatial weight matrix $\tilde{\mathbf{W}}$ towards zero. In other words, the GSTAR( $1 ; 1)$ model is equivalent to the $\operatorname{GSTMA}(n ; 1)$ model.

By using Theorem 2 of every kernel function forming the weight matrix $\overline{\mathbf{w}}$ will produce a convergence rate differently. The convergence rate resulted in the discovery of a finite value $n$ which is an orde of the GSTMA model. The value $n$ for each kernel function with an error of 0.001 can be seen in Figure 2. From Figure 2, it can be classified into 3 groups based on the size of $n$, such as the group $1 \leq n \leq 15,16 \leq n \leq 30$, and $n>30$. On the group $1 \leq n \leq 15$ applies to the uniform kernel function, $n=11$ and the Gaussian, $n=9$. The functions describe that the convergence reached is relatively fast to head zero. The next group is $16 \leq n \leq 30$ satisfied by the Epanechnikov kernel function, $n=25$ and the Cosinus, $n=30$. Both groups can be categorized into finite $n$, but the triangular kernel function, $n$ 
$=688$, can be said $n \rightarrow \infty$. It caused by the triangular function containing a differentiable absolute value function, therefore that it takes time to get convergence.

Each kernel function raised to the power of $\mathrm{n}$ forms a geometric sequence. As a result, the ratio of the Gaussian function becomes the smallest, therefore, that the convergence is also faster. The next smallest ratio in a row is the kernel function of the uniform, the Epanechnikov, the Cosine, and the Triangular. It proved that the invertibility property of the model GSTAR $(1 ; 1)$ can approached by using the GSTMA( $n ; 1)$ model with $n<\infty$. Some error values can be seen in Table 3 .
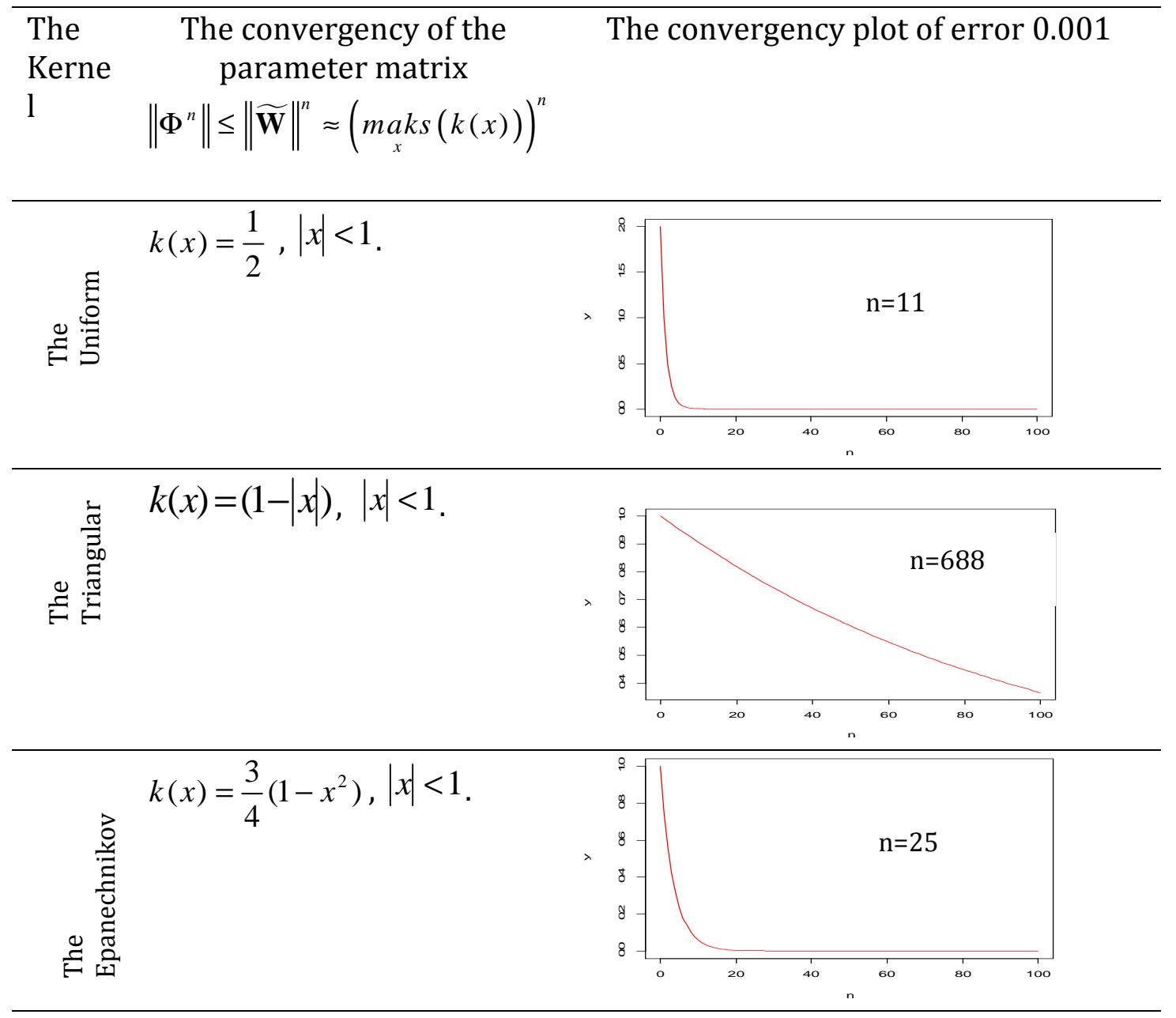

$$
k(x)=\frac{\pi}{4} \operatorname{Cos} \frac{\pi}{2} x,|x|<1
$$

$$
\text { 胥 } \quad k(x)=\frac{\exp \left(-\frac{x^{2}}{2}\right)}{(2 \pi)^{1 / 2}}, x \in \mathrm{R} .
$$

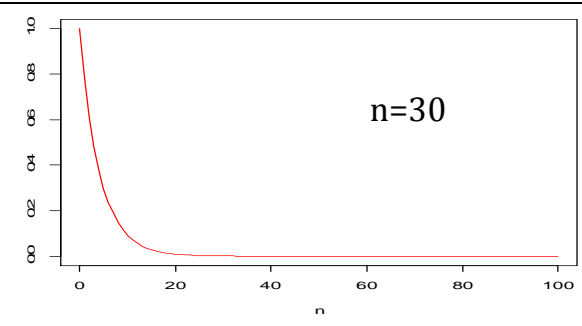


Note: for $x=\frac{\bar{Y}_{i}-\bar{Y}_{j}}{h}$

Figure 2. The order value of the GSTMA model is equivalent to the GSTAR(1;1) model for every single kernel function and the error 0.001 .

Table 3. The finite order of the GSTMA model satisfied the invertibility property of the model GSTAR $(1 ; 1)$

\begin{tabular}{lccc}
\hline \multirow{2}{*}{ The Kernel } & \multicolumn{3}{c}{ The Order of the model GSTMA on some errors } \\
\cline { 2 - 4 } & 0.01 & 0.001 & 0.0001 \\
\hline The Uniform & 8 & 11 & 14 \\
The Triangular & 459 & 688 & 917 \\
The & 17 & 25 & 33 \\
Epanechnikov & & & \\
The Cosinus & 20 & 29 & 39 \\
The Gaussian & 6 & 8 & 11 \\
\hline
\end{tabular}

\section{Case Study}

The GSTAR(1;1) model with the kernel weight will be applied to the tea production data in the 6 plantation field in West Java. The data plot can be seen on Figure 3. The Figure 3 presents the modelling data with time $\mathrm{T}=200$ by 6 observation locations. The Plot of each location shows the data stationary has not been fulfilled (weak stationary), so it is necessary to doing differencing of the data.
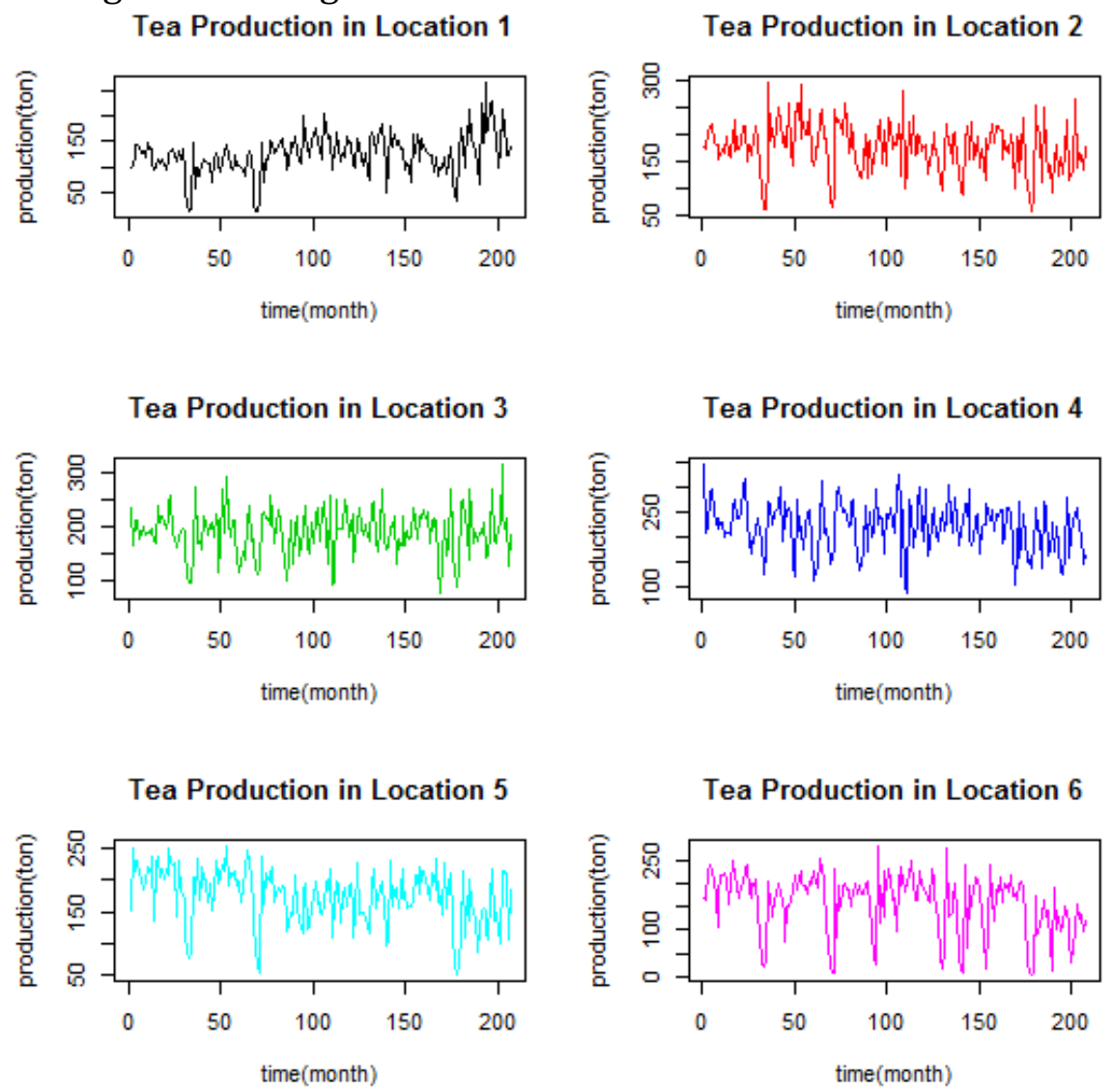

Figure 3. The data Plot of each location. The plot illustrates the data is under weak stationary condition and needs differencing so that the data plot is stationary 
The data is stationary to the mean dan variance after passing once differencing process. The modelling carried out in this paper is the GSTAR(1;1) model. It does not need to identify model. The next step is to determine the spatial weight matrix using the Gaussian kernel (Table 1.) with the optimum bandwith value. This spatial weight matrix is random because it uses the function of tea plantation random variable from each the plantation area. The spatial weight matrix with the Gaussian kernel is represented as following.

$$
\widetilde{W}=\left[\begin{array}{cccccc}
0 & 0.25 & 0.27 & 0.10 & 0.27 & 0.11 \\
0.21 & 0 & 0.24 & 0.16 & 0.23 & 0.16 \\
0.23 & 0.24 & 0 & 0.14 & 0.25 & 0.14 \\
0.12 & 0.22 & 0.18 & 0 & 0.17 & 0.32 \\
0.24 & 0.24 & 0.25 & 0.13 & 0 & 0.13 \\
0.12 & 0.22 & 0.17 & 0.32 & 0.17 & 0
\end{array}\right]
$$

The next step is to determine the parameter value through the least square estimation method and the parameter significance by considering the eigen value obtained of the parameter matrix of the GSTAR(1;1) model. The value of parameter estimation with its validation can be seen on Table 4 .

Table 4. The result of paremeter estimation using least square method and its validation

\begin{tabular}{cccc}
\hline $\begin{array}{c}\text { The parameter of } \\
\text { each location }\end{array}$ & $\begin{array}{c}\text { The value of } \\
\text { parameter } \\
\text { estimation }\end{array}$ & $\begin{array}{c}\text { Confidence Interval } \\
95 \%\end{array}$ & $\begin{array}{c}\text { Validation } \\
\text { (The eigen value of } \\
\text { parameter matrix }<1)\end{array}$ \\
\hline Phi01; Phi 11 & $-0.32 ; 0.01$ & $(-0.37 ;-0.27) ;(-0.03 ; 0.06)$ & Valid \\
Phi02; Phi12 & $-0.48 ; 0.34$ & $(-0.52 ;-0.44) ;(0.27 ; 0.40)$ & Valid \\
Phi03; Phi13 & $-0.56 ; 0.59$ & $(-0.60 ;-0.51) ;(0.53 ; 0.66)$ & Valid \\
Phi04; Phi 14 & $-0.30 ; 0.37$ & $(-0.34 ;-0.27) ;(0.33 ; 0.42)$ & Valid \\
Phi05; Phi15 & $-0.49 ; 0.25$ & $(-0.54 ;-0.45) ;(0.19 ; 0.30)$ & Valid \\
Phi06; Phi16 & $-0.37 ; 0.18$ & $(-0.40 ;-0.34) ;(0.13 ; 0.22)$ & Valid \\
\hline
\end{tabular}

The result of the parameter estimation of the GSTAR(1;1) model with the Gaussian kernel weight is shown as below,

$$
\widehat{\mathbf{Y}}(t)=\left(\widehat{\boldsymbol{\Phi}}_{0}+\widehat{\boldsymbol{\Phi}}_{1} \widetilde{\mathbf{W}}\right) \mathbf{Y}(t-1)
$$

with

$$
\begin{aligned}
\widehat{\boldsymbol{\Phi}}_{0}= & {\left[\begin{array}{cccccc}
-0.32 & 0 & 0 & 0 & 0 & 0 \\
0 & -0.48 & 0 & 0 & 0 & 0 \\
0 & 0 & -0.56 & 0 & 0 & 0 \\
0 & 0 & 0 & -0.30 & 0 & 0 \\
0 & 0 & 0 & 0 & -0.49 & 0 \\
0 & 0 & 0 & 0 & 0 & -0.37
\end{array}\right] \text { and } } \\
\widehat{\boldsymbol{\Phi}}_{1} & =\left[\begin{array}{cccccc}
0.01 & 0 & 0 & 0 & 0 & 0 \\
0 & 0.34 & 0 & 0 & 0 & 0 \\
0 & 0 & 0.59 & 0 & 0 & 0 \\
0 & 0 & 0 & 0.37 & 0 & 0 \\
0 & 0 & 0 & 0 & 0.25 & 0 \\
0 & 0 & 0 & 0 & 0 & 0.18
\end{array}\right]
\end{aligned}
$$


The data Plot estimated from each location with the GSTAR(1;1) model can be viewed in Figure 4 with its RMSE value respectively. It concludes that the estimation value to follow the original value pattern and the RMSE value is quite small.

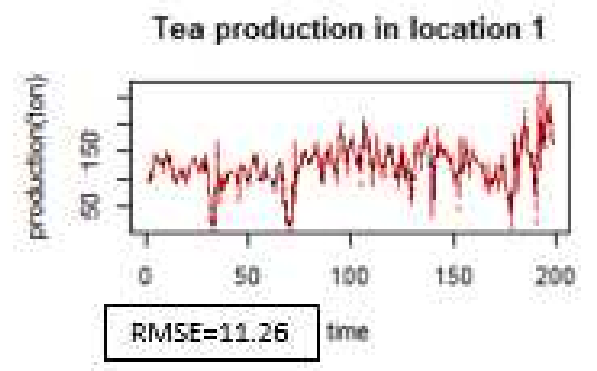

Tea production in location 3

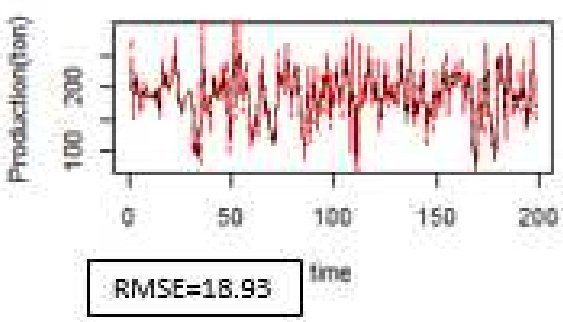

Tea production in location 5

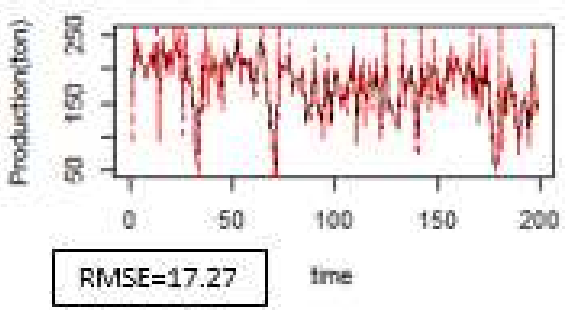

Tea production in location 2

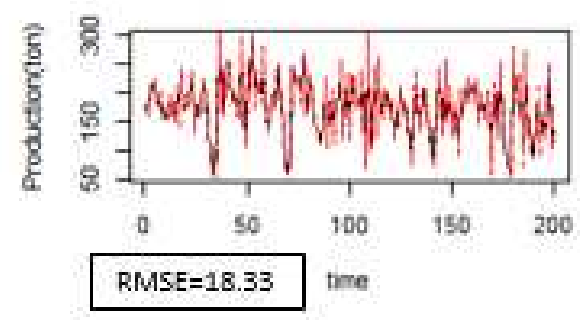

Tea production in location 4

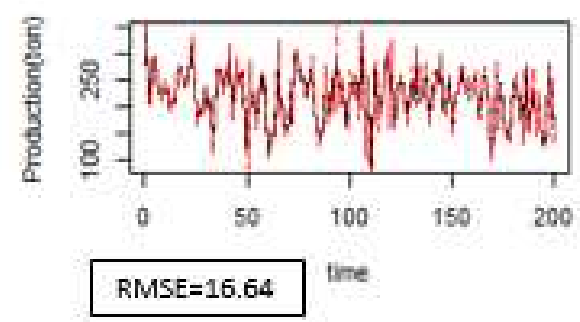

Tea production in location 6

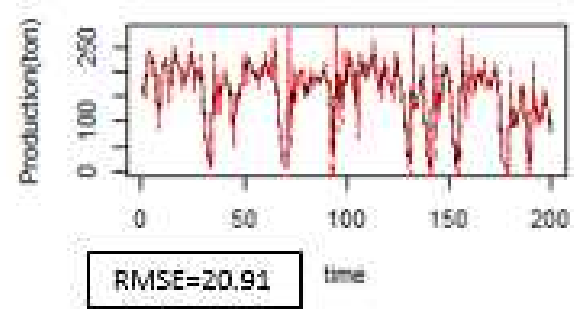

Figure 4. The plot of the both estimation and original value of the GSTAR(1;1) model with the Gaussian kernel weight. The black line presents the original value and the red line is the estimation value.

The residual test of this model can be viewed in Figure 5. The residual scatter plot and QQ plot show that the assumptions of randomness and normality are met. 

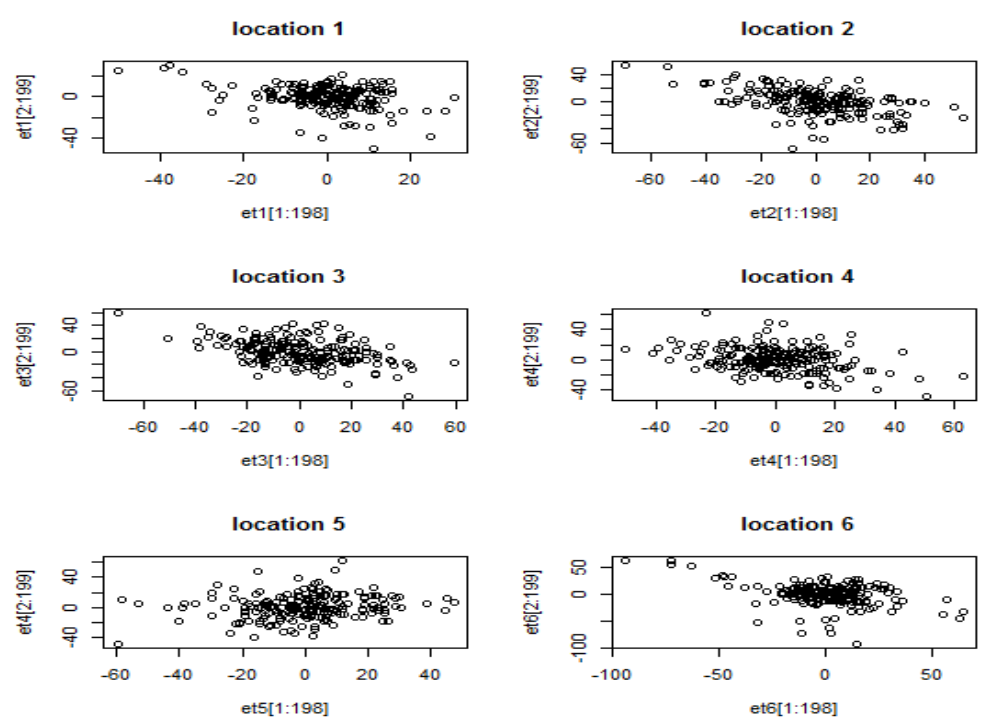

(a)
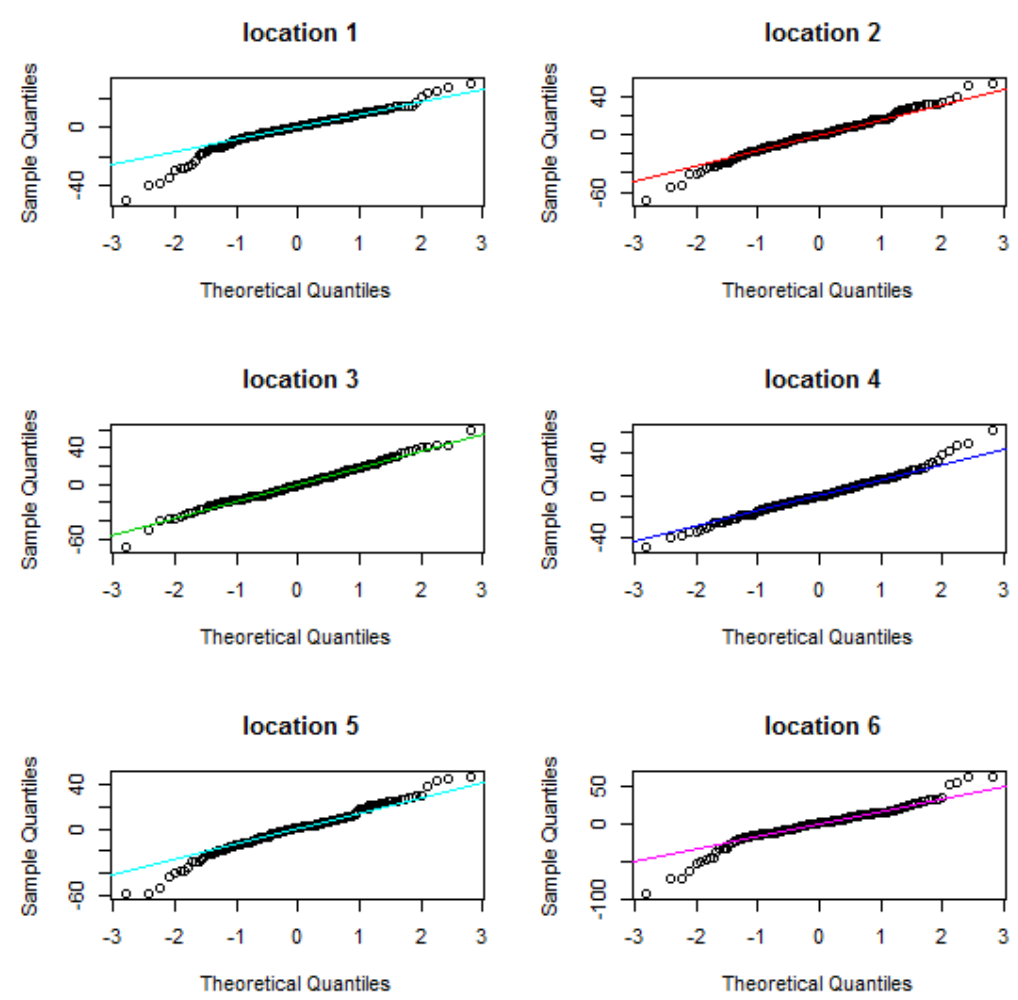

(b)

Figure 5. The results of the residual test plot that meet the assumption of randomness (a) and normality (b)

\section{CONCLUSION}

The use of the kernel weight matrix also affects the invertibility property of the model $\operatorname{GSTAR}(1 ; 1)$ to the order of the GSTMA $(\infty, 1)$. The result obtained is the time order of the finite orde $7<n<30$. On the Triangular kernel resulted in the relatively great value $n$, so that it does not apply to the kernel with a finite value $n$. The model implementation of the tea production data over 6 plantation area in West Java can be applied to this research. 
This is because the spatial weight to use production data applied to the Gaussian kernel function according to the data description from each research location.

\section{REFERENCES}

[1] G. Box, G. Jenkins and G.Reinsel, Time Series Analysis, forecasting and Control, 3rd edition, New Jersey: Prentice Hall, 1994.

[2] R. Tsay, Multivariate Time Series Analysis With R and Financial Application, New Jersey: John Willey \& Sons, 2014.

[3] Y. Yundari, U. S. Pasaribu, U. Mukhaiyar and M.N.Heriawan, "Spatial Weight Determination of GSTAR(1;1) Model by Using Kernel Function," in IOP Conf, Series: Journal of Physics , 2018.

[4] Y. Yundari, U. Pasaribu and U.Mukhaiyar, "Assumption Errors of Generalized STAR Model," J. Math. Fund. Sci., vol. 49, no. 2, pp. 136-155, 2017.

[5] U. Mukhaiyar and U.S.Pasaribu, "A New Procedure of Generalized STAR Modelling using IAcM Approach," ITB J. Sci., vol. 44A, no. 2, pp. 179-192, 2012.

[6] S. Borovkova, B. Ruchjana and H. Lopuhaa, "Least Squares Estimation of Generalized Space Time AutoRegressive (GSTAR) Model and Its Properties," in AIP Conf. Proc. , 2012.

[7] D. Masteriana, M. Riani and U.Mukhaiyar, "Generalized STAR(1;1) with outlier-Case Study of Begal in Medan, North Sumatera," in J.Phys.:Conf. Ser., 2019.

[8] H. Bonar, B. Ruchjana and G. Darmawan, "Development of generalized space time autoregressive integrated with ARCH galat (GSTARI-ARCH) model based on consumer price index phenomenon at several cities in North Sumatera province," in AIP Conference Proceedings, 2013.

[9] A. Iriany, Suhariningsih, B. Ruchjana and Setiawan, "Prediction of Precipitation Data at Batu Town using the GSTAR(1,p)-SUR Model," Journal of Basic and Applied Scietific Research, vol. 3, no. 1, pp. 860-865, 2013.

[10] A. S. Abdullah, S. Matoha, D. A. Lubis, A. N. Falah, M. Jaya, E. Hermawan and B. N. R. B, "Implementation of Generalized Space Time Autoregressive (GSTAR)-Kriging Model for Predicting Rainfall Data at Unobserved Locations in West Java," Applied Mathematics \& Information Sciences, vol. 12, no. 3, pp. 607-615, 2018.

[11] N. Nurhayati, U. S. Pasaribu and O. Neswan, "Application of Generalized Space-time Autoregressive Model on GDP Data in West European Countries," Journal of Probability and Statistics, vol. 2012, 2012.

[12] Suhartono and Subanar, "The Optimal Determination of Space Weight in GSTAR Model by using Cross-correlation Inference," Quantitativ Methods, vol. 2, no. 2, pp. 45-53, 2006.

[13] R. Nugraha, S. Setyowati, U. Mukhaiyar and A. Yuliawati, "Prediction of Oil Palm Production using the Weight Average of Fuzzy Sets Concept Approach," in AIP Conference Proceeding, Bandung, 2015.

[14] Yundari, N. Huda, U. Pasaribu, U. Mukhaiyar and K. Sari, "Stationary process in GSTAR(1;1) through kernel function approach," in AIP Conference Proceeding, Pontianak, 2020. 
[15] D. U. Wutsqa and Suhartono, "Seasonal Multivariate Time Series Forecasting on Tourism Data by using VAR-GSTAR Model," Jurnal Ilmu Dasar, vol. 11, no. 1, pp. 101109, 2010.

[16] N. Fadlilah, U. Mukhiayar and F. Fahmi, "The Generalized STAR(1;1) Modeling with Time Correlated Galats to Red Chili Weekly Prices of some Traditional Markets in Bandung," in AIP Conference Proceeding, Bandung, 2015.

[17] M. Wand and M. Jones, Kernel Smoothing, New York: Springer- Science+Business Media B.V, 1995.

[18] M. Michalack, "Time series pediction with periodic kernels," Pattern Analitic Application, vol. 14, no. 0, pp. 283-293, 2011.

[19] R. Horn and C. Johnson, Matrix analysis, 2nd, New York: Cambridge Univesity Press, 2013. 